\title{
Black Holes in General Relativity
}

\author{
S. W. HAWKING \\ Institute of Theoretical Astronomy, University of Cambridge, Cambridge, England
}

Received October 15, 1971

\begin{abstract}
It is assumed that the singularities which occur in gravitational collapse are not visible from outside but are hidden behind an event horizon. This means that one can still predict the future outside the event horizon. A black hole on a spacelike surface is defined to be a connected component of the region of the surface bounded by the event horizon. As time increase, black holes may merge together but can never bifurcate. A black hole would be expected to settle down to a stationary state. It is shown that a stationary black hole must have topologically spherical boundary and must be axisymmetric if it is rotating. These results together with those of Israel and Carter go most of the way towards establishing the conjecture that any stationary black hole is a Kerr solution. Using this conjecture and the result that the surface area of black holes can never decrease, one can place certain limits on the amount of energy that can be extracted from black holes.
\end{abstract}

\section{Introduction}

It has been known for some time that a non-rotating star of more than about two solar masses has no low temperature equilibrium configuration. This means that such a star must undergo catastrophic collapse when it has exhausted its nuclear fuel unless it has managed to eject sufficient matter to reduce its mass to less than twice that of the sun. If the collapse is exactly spherically symmetric, the metric is that of the Schwarzschild solution outside the star and has the following properties (see Fig. 1):

1. The surface of the star will pass inside the Schwarzschild radius $r=2 G c^{-2} M$. After this has happened there will be closed trapped surfaces $[1,2]$ around the star. A closed trapped surface is a spacelike 2-surface such that both the future directed families of null geodesics orthogonal to it are converging. In other words, it is in such a strong gravitational field that even the outgoing light from it is dragged inwards.

2. There is a space-time singularity.

3. The singularity is not visible to observers who remain outside the Schwarzschild radius. This means that the breakdown of our present physical theory which one expects to occur at a singularity cannot affect 
what happens outside the Schwarzschild radius and one can still predict the future in the exterior region from Cauchy data on a spacelike surface.

One can ask whether these three properties of spherical collapse are stable, i.e. whether they would still hold if the initial data for the collapse were perturbed slightly. This is vital because no real collapse situation will ever be exactly spherical. From the stability of the Cauchy problem in general relativity [3] one can show that a sufficiently small perturbation of the initial data on a spacelike surface will produce a perturbation of the solution which will remain small on a compact region in the Cauchy development of the surface. This shows that property (1) is stable, since there is a compact region in the Cauchy development of the initial surface which contains closed trapped surfaces. It then follows that property (2) is stable provided one makes certain reasonable assumptions such as that the energy density of matter is always positive. This is because the existence of a closed trapped surface implies the occurrence of a singularity under these conditions [4]. There remains the problem of the stability of property (3). Since the question of whether singularities are visible from outside depends on the solution at arbitrarily large times, one cannot appeal to the result on the stability of the Cauchy problem referred to above. Nevertheless it seems a reasonable conjecture that property (3) is indeed stable. If this is the case, we can still predict what happens outside collapsed objects, and we need not worry that something unexpected might occur every time a star in the galaxy collapsed. My belief in this conjecture is strengthened by the fact that Penrose [5] has tried and failed to obtain a contradiction to it, which would show that naked singularities must occur. Penrose's method has been generalised by Gibbons [6] who has shown that it cannot lead to a contradiction at least in some cases. This paper will be written therefore on the assumption that property (3) holds.

In Section 2 a black hole is defined in terms of a event horizon and it is shown that the surface area of a black hole cannot decrease with time. In Section 3 it is shown that a rotating stationary black hole must be axisymmetric, and in Section 4 it is shown that any stationary black hole must have a topologically spherical boundary. Together with the results of Israel and Carter, this strongly supports the conjecture that a black hole settles down to a Kerr solution. This conjecture is used in Section 5 to relate the surface area of a black hole to its mass, angular momentum and electric charge. Using the result that the surface area cannot decrease with time one can then place upper bounds on the amounts of energy that can be extracted from black holes. These limits suggest that there may be a spin dependent force between two black holes analogous to that between magnetic dipoles. 


\section{The Event Horizon}

In order to discuss the region outside a collapsed object one needs a precise notion of infinity in an asymptotically flat space-time. This is provided by Penrose's concept of a weakly asymptotically simple space [2]; the spacetime manifold $\mathscr{M}$ of such a space can be imbedded in a larger manifold $\tilde{\mathscr{M}}$ on which there is a Lorentz metric $\tilde{g}_{a b}$ which is conformal to the spacetime metric $g_{a b}$, i.e. $\tilde{g}_{a b}=\Omega^{2} g_{a b}$ where $\Omega$ is a smooth function which is zero and has non-vanishing gradient on the boundary of $\mathscr{M}$ in $\tilde{\mathscr{M}}$. This boundary consists of two null hypersurfaces $\mathscr{I}^{+}$and $\mathscr{I}^{-}$ which each have topology $S^{2} \times R^{1}$ and which represent future and past null infinity respectively. One can then interpret property (3) as saying that it should be possible to predict events near $\mathscr{I}^{+}$. I shall therefore say that a weakly asymptotically simple space is (future) asymptotically predictable if there is a partial Cauchy surface $\mathscr{S}$ such that $\mathscr{I}^{+}$lies in the closure in $\tilde{\mathscr{M}}$ of $D^{+}(\mathscr{S})$, the future Cauchy development of $\mathscr{S}$. (A partial Cauchy surface is a spacelike surface without edge which does not intersect any non-spacelike curve more than once. $D^{+}(\mathscr{S})$ is the set of all points $p$ such that every past directed non-spacelike curve from $p$ intersects $\mathscr{S}$ if extended far enough.)

Roughly speaking one would expect a space to be asymptotically predictable if there are no singularities in $J^{+}(\mathscr{S})$, the future of $\mathscr{S}$, which are naked, i.e. which lie in $J^{-}\left(\mathscr{I}^{+}\right)$, the past of future null infinity. One can make this more precise. Consider an asymptotically predictable space in which there are no singularities to the past of $\mathscr{Y}$. Suppose there is a closed trapped surface $\mathscr{T}$ in $D^{+}(\mathscr{S})$. Then there will be a singularity to the future of $\mathscr{T}$, i.e. there will be a nonspacelike geodesic in $J^{+}(\mathscr{T})$ which is future incomplete. Can this geodesic be seen from $\mathscr{I}^{+}$? The answer is no. For suppose $\mathscr{T}$ intersected $J^{-}\left(\mathscr{I}^{+}\right)$. Then there would be a point $p \in \mathscr{I}^{+}$in $J^{+}(\mathscr{T})$. The past directed null geodesic generator of $\mathscr{I}^{+}$through $p$ would eventually leave $J^{+}(\mathscr{T})$ and so would contain a point $q$ of the boundary $\dot{J}^{+}(\mathscr{T})$. Now the boundary of the future of any closed set $\mathscr{W}$ is generated by null geodesic segments which either have no past end-points or have past end-points on $\mathscr{W}[2,4]$. Since the generator $\lambda$ of $\dot{J}^{+}(\mathscr{T})$ through $q$ would enter $D^{+}(\mathscr{S})$ it would have to have an end point on $\mathscr{T}$ since otherwise it would intersect $\mathscr{S}$ and pass into the past of $\mathscr{S}$ which would be impossible, as $\mathscr{T}$ is to the future of $\mathscr{S}$. The generator $\lambda$ would intersect $\mathscr{T}$ orthogonally. However, as $\mathscr{T}$ is a closed trapped surface, the null geodesics orthogonal to $\mathscr{T}$ are converging. Together with the weak energy condition: $T_{a b} K^{a} K^{b} \geqq 0$ for any timelike vector $K^{a}$, this implies that there will be a point conjugate to $\mathscr{T}$ within a finite affine length on any null geodesic orthogonal to $\mathscr{T}[4]$. Points on such a geodesic beyond the conjugate point will lie in 


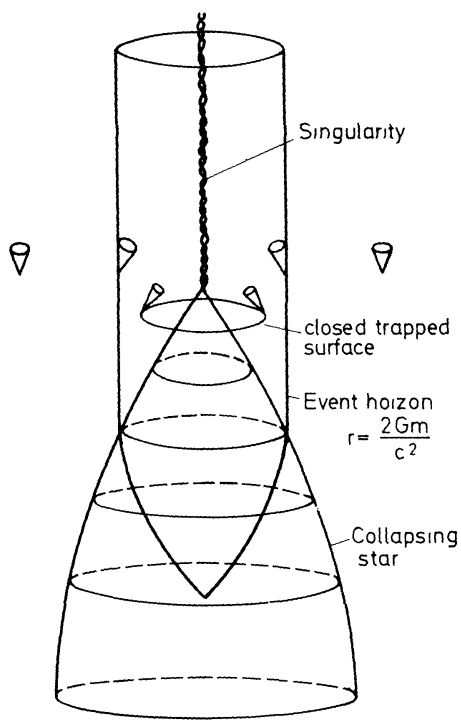

Fig. 1. Spherical collapse

the interior of $J^{+}(\mathscr{T})$ and not on its boundary $[2,4]$. However the generator $\lambda$ of $\dot{J}^{+}(\mathscr{T})$ would have infinite affine length from $\mathscr{T}$ to $\mathscr{I}^{+}$ since $\mathscr{I}^{+}$is at infinity. This establishes a contradiction which shows that $\mathscr{T}$ does not intersect $J^{-}\left(\mathscr{I}^{+}\right)$. Thus the future incomplete geodesic in $J^{+}(\mathscr{T})$ is not visible from $\mathscr{I}^{+}$.

Since $J^{-}\left(\mathscr{I}^{+}\right)$does not contain $\mathscr{T}$, its boundary $\dot{J}^{-}\left(\mathscr{I}^{+}\right)$must be nonempty. This is the event horizon for $\mathscr{I}^{+}$and is the boundary of the region from which particles or photons can escape to infinity. It is generated by null geodesic segments which have no future end-points. The convergence $\varrho$ of these generators cannot be positive. For suppose it were positive on some open set $\mathscr{U}$ of $\dot{J}^{-}\left(\mathscr{I}^{+}\right)$. Let $\mathscr{F}$ be a spacelike 2-surface in $\mathscr{U}$. Then the outgoing null geodesics orthogonal to $\mathscr{F}$ would be converging. One could deform a small part of $\mathscr{F}$ so that it intersected $J^{-}\left(\mathscr{I}^{+}\right)$but so that the outgoing null geodesics orthogonal to $\mathscr{F}$ were still converging. This again would lead to a contradiction since the null geodesics orthogonal to $\mathscr{F}$ could not remain in $\dot{J}^{+}(\mathscr{F})$ all the way out to $\mathscr{I}^{+}$.

If there were a point on the event horizon which was not in $D^{+}(\mathscr{S})$, the future Cauchy development of $\mathscr{S}$, a small perturbation could result in there being points near $\mathscr{I}^{+}$which were not in $D^{+}(\mathscr{S})$. Since I am assuming that asymptotic predictability is stable, I shall slightly extend the definition to exclude this kind of situation. In an asymptotically 
predictable space $J^{+}(\mathscr{S}) \cap J^{-}\left(\mathscr{I}^{+}\right)$is in $D^{+}(\mathscr{S})$. I shall say that such a space is strongly asymptotically predictable if in addition $J^{+}(\mathscr{S}) \cap \dot{J}\left(\mathscr{I}^{+}\right)$ is in $D^{+}(\mathscr{S})$.

In such a space one can construct a family $\mathscr{S}(t)(t>0)$ of partial Cauchy surfaces in $D^{+}(\mathscr{S})$ such that

(a) for $t_{2}>t_{1}, \mathscr{S}\left(t_{2}\right) \subset J^{+}\left(\mathscr{S}\left(t_{1}\right)\right)$;

(b) each $\mathscr{S}(t)$ intersects $\mathscr{I}^{+}$in a 2 -sphere $\mathscr{A}(t)$;

(c) for each $t>0, \mathscr{S}(t) \cup\left[\mathscr{I}^{+} \cap J^{-}(\mathscr{A}(t))\right]$ is a Cauchy surface for $D^{+}(\mathscr{S})$.

The construction is as follows. Choose a suitable family $\mathscr{A}(t)$ of 2 -spheres on $\mathscr{I}^{+}$. Put a volume measure on $\mathscr{M}$ so that the total volume of $\mathscr{M}$ in this measure is finite [7]. Define the functions $f(p)$ and $h(p, t), p$ $\in D^{+}(\mathscr{S})$ as the volumes of $J^{+}(p) \cap D^{+}(\mathscr{S})$ and $\left[J^{-}(p)-J^{-}(\mathscr{A}(t))\right] \cap D^{+}(\mathscr{S})$ respectively. They will be continuous in $p$ and $t$. The surface $\mathscr{S}(t)$ is then defined to be the set of points $p$ such that $h(p, t)=t f(p)$.

For sufficiently large $t$, the surfaces $\mathscr{S}(t)$ will intersect the event horizon and so $\mathscr{B}(t)$ defined as $\mathscr{S}(t)-J\left(\mathscr{I}^{+}\right)$will be nonempty. I shall define a black hole on the surface $\mathscr{S}(t)$ to be a connected component of $\mathscr{B}(t)$. In other words, it is a region of $\mathscr{S}(t)$ from which there is no escape to $\mathscr{I}^{+}$. As time increases, black holes may merge together and new black holes may be created by further bodies collapsing but a black hole can never bifurcate. For suppose the black hole $\mathscr{B}_{1}\left(t_{1}\right)$ on the surface $\mathscr{S}\left(t_{1}\right)$ divided into two black holes $\mathscr{B}_{2}\left(t_{2}\right)$ and $\mathscr{B}_{3}\left(t_{2}\right)$ by a later surface $\mathscr{S}\left(t_{2}\right)$. Then $\mathscr{B}_{2}\left(t_{2}\right)$ and $\mathscr{B}_{3}\left(t_{2}\right)$ would each have to contain points of $J^{+}\left(\mathscr{B}_{1}\left(t_{1}\right)\right)$. However every nonspacelike curve which intersected $\mathscr{B}_{1}\left(t_{1}\right)$ would intersect $\mathscr{S}\left(t_{2}\right)$. Therefore $J^{+}\left(\mathscr{B}_{1}\left(t_{1}\right)\right) \cap \mathscr{S}\left(t_{2}\right)$ would be connected and would be contained in $\mathscr{B}_{2}\left(t_{2}\right) \cup \mathscr{B}_{3}\left(t_{2}\right)$.

Suppose that $\mathscr{M}$ is initially nonsingular in the sense that $J^{-}(\mathscr{S})$, the region to the past of $\mathscr{S}$, is isometric to a region to the past of a Cauchy surface in an asymptotically simple space [2]. Then for small values of $t$, the surfaces $S(t)$ will be compact. However the surfaces $\mathscr{S}(t)$ are homeomorphic to each other for all $t>0$ and so they will all be compact. This implies that the boundary $\partial \mathscr{B}_{1}(t)$ of a black hole $\mathscr{B}_{1}(t)$ will be compact.

Since the generators of $\dot{J}^{-}\left(\mathscr{I}^{+}\right)$have no future end points and have convergence $\varrho \leqq 0$, the surface area of $\partial \mathscr{B}_{1}(t)$ cannot decrease with $t$. If two black holes $\mathscr{B}_{1}\left(t_{1}\right)$ and $\mathscr{B}_{2}\left(t_{1}\right)$ on the surface $\mathscr{S}\left(t_{1}\right)$ merge to form a single black hole $\mathscr{B}_{3}\left(t_{2}\right)$ on a later surface $\mathscr{S}\left(t_{2}\right)$, then the area of $\partial \mathscr{B}_{3}\left(t_{1}\right)$ must be at least the sum of the areas of $\partial \mathscr{B}_{1}\left(t_{1}\right)$ and $\partial \mathscr{B}_{2}\left(t_{1}\right)$. In fact it must be strictly greater than this sum because $\partial \mathscr{B}_{3}\left(t_{2}\right)$ contains two disjoint closed sets which correspond to the generators of $\dot{J}\left(\mathscr{I}^{+}\right)$which intersect $\partial \mathscr{B}_{1}\left(t_{1}\right)$ and $\partial \mathscr{B}_{2}\left(t_{1}\right)$. Since $\partial \mathscr{B}_{3}\left(t_{2}\right)$ is connected, it must also contain an open set of points which correspond to generators which 
have past end points between $t_{2}$ and $t_{1}$. These results will be used in the next section to place certain limits on the possible behaviour of black holes.

\section{Stationary Black Holes}

In a collapse that was strongly asymptotically predictable one would expect the solution outside the event horizon to become stationary eventually. This suggests that one should study exactly stationary solutions containing black holes in the hope that one of these will represent the final state of a collapsed system. In this section I shall therefore consider spaces which satisfy the following conditions:

(i) They are weakly asymptotically simple.

(ii) There exists a one parameter isometry group $\phi_{t}: \mathscr{M} \rightarrow \mathscr{M}$ whose Killing vector $K^{a}$ is timelike near $\mathscr{I}^{+}$and $\mathscr{I}^{-}$.

(iii) There exist both a past event horizon $\dot{J}^{+}\left(\mathscr{I}^{-}\right)$and a future event horizon $\dot{J}^{-}\left(\mathscr{I}^{+}\right)$.

(iv) There exists a partial Cauchy surface $\mathscr{S}$ from which the exterior region $\bar{J}^{+}\left(\mathscr{I}^{-}\right) \cap \bar{J}^{-}\left(\mathscr{I}^{+}\right)$can be determined, i.e. the exterior region is contained in $D^{+}(\mathscr{S}) \cup D^{-}(\mathscr{S})$, the Cauchy development of $\mathscr{S}$.

(v) The two event horizons $\dot{j}^{+}\left(\mathscr{I}^{-}\right)$and $\dot{j}^{-}\left(\mathscr{I}^{+}\right)$intersect in a compact surface $\mathscr{F}$.

In a real situation where an initially nonsingular body collapses there will not of course be any past event horizon, since that part of the space will be inside the body and so will be different and will be nonstationary. The statement that a past horizon exists is therefore to be understood as a condition on the analytic continuation of the stationary solution to which the real solution tends. Except in certain limiting cases, one can prove that provided there are no singularities in the exterior region, there will indeed be a past horizon which will intersect the future horizon. Penrose however has questioned whether it is reasonable to assume the nonexistence of naked singularities in the unphysical past region of the analytic extension. In fact the results that will be given in this paper can be obtained without assuming anything about the behaviour of the solution in the past though the proofs are then considerably more complicated. They will be given in [3].

Israel [8] has shown that a space which satisfies conditions (i)-(v) must be the Schwarzschild solution if it also satisfies the following conditions:

(vi) It is empty, i.e. $T_{a b}=0$.

(vii) It is static and not merely stationary, i.e. the Killing vector $K^{a}$ is hypersurface orthogonal so $\eta^{a b c d} K_{b} K_{c ; d}=0$. 
(viii) The gradient of $K^{a} K_{a}$ is not zero anywhere outside the horizon. This means that there is no neutral point at which a particle can remain at rest outside the black hole.

Carter [9] has considered spaces which satisfy conditions (i)-(vi) and

(ix) are axisymmetric.

(x) The surface $\mathscr{F}$ which is the intersection of the two horizons has the topology $S^{2}$.

He shows that such spaces fall into disjoint families, each depending on at most two parameters $m$ and $a$. The parameter $m$ represents the mass of the black hole as measured from infinity and the parameter $a$ is $h c / G m$ where $h$ is the angular momentum as measured from infinity. One such family is known, namely the Kerr solutions with $a^{2}<m^{2}$ [10]. It seems unlikely that there are any others. It has therefore been conjectured that, at least in a collapse that does not deviate too much from spherical symmetry, the solution outside the horizon tends to one of the Kerr solutions with $a^{2}<m^{2}$. I shall here give further support to this conjecture by justifying condition (ix), that is, I shall show that a rotating black hole must be axisymmetric if it satisfies conditions (i)-(v). Condition (x) will be justified in the next section.

I shall assume that the matter in the space satisfies the weak energy condition and obeys well behaved hyperbolic equations such as Maxwell's equations or those for a scalar field. It then follows that the solution must be analytic near infinity where the Killing vector is timelike [11]. I shall take the solution elsewhere to be the analytic continuation of this region near infinity. The idea is now to consider the solution immediately to the future of the surface $F$ of intersection of the two horizons (Fig. 2). This is determined by Cauchy data on the two horizons. By analyticity the solution in this region determines the local nature of the solution everywhere else. I shall show that the Cauchy data on the intersecting horizons is invariant under a continuous group which leaves the intersection surface $\mathscr{F}$ invariant but moves points on one horizon along the null generators of the horizon towards $\mathscr{F}$ and moves points on the other horizon along the generators away from $\mathscr{F}$ (Fig. 3). From the uniqueness of the Cauchy problem it then follows that the solution must admit a Killing vector field which on the horizon is directed along the null generators. However, in general, the Killing vector field $K^{a}$ which is timelike near infinity will be spacelike on the horizon. There must thus be two independent Killing vector fields and a two parameter isometry group. Near infinity the extra symmetry will have the character of a Poincaré transformation. Since a black hole in asymptotically flat space is not invariant under a space translation or a Lorentz boost, the extra symmetry must correspond to a spatial rotation and so the solution will 


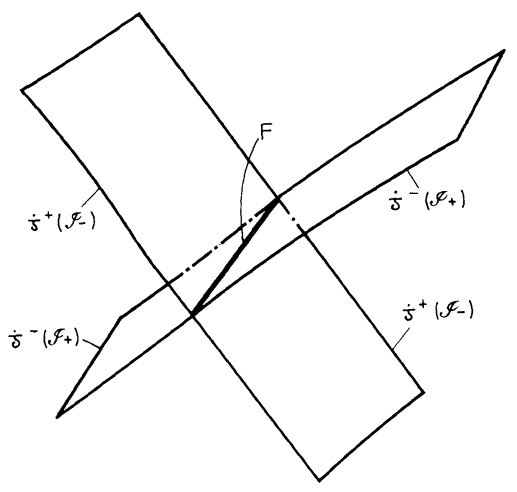

Fig. 2. The intersecting horizons

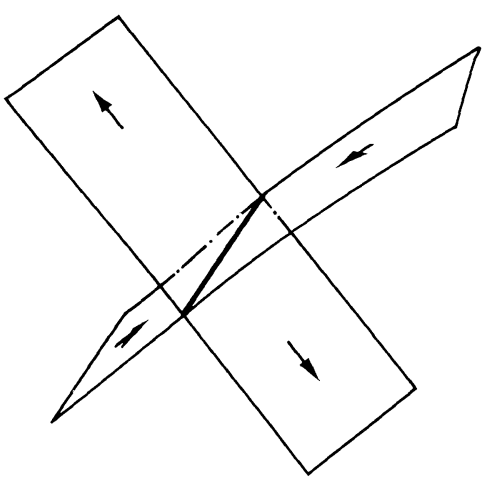

Fig. 3. The transformation $\theta_{u}$

be axisymmetric. Carter [12] has shown that in a stationary axisymmetric asymptotically flat space, the two Killing vectors must commute.

To show that the Cauchy data on the horizons is invariant under a group, I shall first show that the null generators of the horizons have zero convergence $\varrho$ and shear $\sigma$ (see [13] for definitions). For this purpose consider a spacelike 2 -sphere $\mathscr{C}$ on $\mathscr{I}^{-}$. The family of surfaces $\mathscr{C}(t) \equiv \phi_{t}(\mathscr{C})$ obtained by moving $\mathscr{C}$ along the Killing vector $K^{a}$ will cover $\mathscr{I}^{-}$. Let $\lambda$ be a null geodesic generator of the future horizon $j^{-}\left(\mathscr{I}^{+}\right)$which intersects $\mathscr{F}$. The strong causality condition [14] will hold on the compact set $\mathscr{F}$ since it is in the Cauchy development of $\mathscr{S}$. This means that $\lambda$ must leave $\mathscr{F}$ and so must leave $\dot{J}^{+}\left(\mathscr{I}^{-}\right)$and enter $J^{+}\left(\mathscr{I}^{-}\right)$. Suppose there were some $t^{\prime}$ such that $\lambda$ did not intersect $J^{+}\left(\mathscr{C}\left(t^{\prime}\right)\right)$. Then for any $t$, the 
generator $\phi_{t}(\lambda)$ would not intersect $J^{+}\left(\mathscr{C}\left(t^{\prime}+t\right)\right)$. Since $\mathscr{F}$ is compact there would be a point $q \in \mathscr{F}$ which was a limit point of $\mathscr{F} \cap \phi_{t}(\lambda)$ as $t \rightarrow-\infty$ and there would be a null direction at $q$ which was a limit of the direction of the $\phi_{t}(\lambda)$. The future directed null geodesic from $q$ in this direction would lie in $j^{-}\left(\mathscr{I}^{+}\right)$because $\dot{J}^{-}\left(\mathscr{I}^{+}\right)$is a closed set and its generators have no future end-points. Thus it would enter $J^{+}\left(\mathscr{I}^{-}\right)$. However it could not intersect $J^{+}(\mathscr{C}(t))$ for any $t$ since it was a limit of the $\phi_{t}(\lambda)$ as $t \rightarrow-\infty$. This establishes a contradiction which shows that $\lambda$ must intersect $J^{+}(\mathscr{C}(t))$ for every $t$.

The generators of $\dot{J}^{-}\left(\mathscr{I}^{+}\right)$which intersect $\mathscr{F}$ form a compact set invariant under $\phi_{t}$. Let $\mathscr{L}(t)$ be the 2-surface which is the intersection of these generators with $\dot{J}^{+}(\mathscr{C}(t))$. As $t$ increases, $\mathscr{L}(t)$ will move to the future along the generators of $\dot{J}^{-}\left(\mathscr{I}^{+}\right)$. The convergence $\varrho$ of these generators is less than or equal to zero. If it were less than zero anywhere the area of $\mathscr{L}(t)$ would increase. However the area of $\mathscr{L}(t)$ must remain the same since it is moving under the isometry $\phi_{t}$. Thus $\varrho=l_{a ; b} m^{a} \bar{m}^{b}=0$ where $l^{a}$ is the null tangent vector to the generators of $\dot{J}^{-}\left(\mathscr{I}^{+}\right)$and $m^{a}$ and $\bar{m}^{a}$ are complex conjugate null vectors orthogonal to $l^{a}$ with $m^{a} \bar{m}_{a}$ $=-1$. It then follows from the weak energy condition that the shear $\sigma=l_{a ; b} m^{a} m^{b}$, Ricci tensor component $\Phi_{00}=-\frac{1}{2} R_{a b} l^{a} l^{b}$ and Weyl tensor component $\psi_{0}=-\mathscr{C}_{a b c d} l^{a} m^{b} l^{c} m^{d}$ are zero on $\dot{J}^{-}\left(\mathscr{I}^{+}\right)$[13]. Similarly the corresponding quantities $\mu=-n_{a ; b} \bar{m}^{a} m^{b}, \lambda=-n_{a ; b} \bar{m}^{a} \bar{m}^{b}, \Phi_{22}$ $=-\frac{1}{2} R_{a b} n^{a} n^{b}$ and $\psi_{4}=-C_{a b c d} n^{a} \bar{m}^{b} n^{c} \bar{m}^{d}$ must be zero on $\dot{j}^{+}\left(\mathscr{I}^{-}\right)$where $n^{a}$ is the null vector tangent to the generators of $\dot{J}^{+}\left(\mathscr{I}^{-}\right)$and $m^{a}$ and $\overline{\mathrm{m}}^{a}$ are orthogonal to $n_{a}$. For the empty space Einstein equations the Cauchy data is determined by $\psi_{0}$ on $\dot{J}^{-}\left(\mathscr{I}^{+}\right), \psi_{4}$ on $\dot{J}^{+}\left(\mathscr{I}^{-}\right)$and $\varrho, \mu$ and $\psi_{2}$ $=-\frac{1}{2} C_{a b c d}\left(l^{a} n^{b} l^{c} n^{d}+l^{c} n^{b} m^{c} \bar{m}^{d}\right)$ on the intersection surface $\mathscr{F}[19,16]$. Apart from $\psi_{2}$, these quantities are all zero in the present case. At $\mathscr{F}$ one can normalise $l^{a}$ and $n^{a}$ by $l^{a} n_{a}=1$ and one can then parallelly propagate them and $m^{a}$ and $\bar{m}^{a}$ up the horizons. There remains the freedom $l^{a} \rightarrow e^{u} l^{a}$, $n^{a} \rightarrow e^{-u} n^{a}$ where $u$ is a function on $\mathscr{F}$. If $u$ is not constant on $\mathscr{F}$ the rotation coefficient $\alpha+\bar{\beta}=l_{a ; b} n^{a} \bar{m}^{b}$ will be changed by this transformation. However if $u$ is constant all the rotation coefficients and Riemann tensor components will be unchanged under this transformation if one moves from a point on $\dot{J}^{-}\left(\mathscr{I}^{+}\right)$an affine distance $v$ from $\mathscr{F}$ to a point on the same generator an affine distance $e^{-u} v$ from $\mathscr{F}$. Similarly everything is unchanged on $j^{+}\left(\mathscr{I}^{-}\right)$if one moves from an affine distance of $w$ to one of $e^{u} w$. This means that the Cauchy data on the horizon is invariant the one parameter transformation $\theta_{u}$ which leaves $\mathscr{F}$ invariant and is generated by the vector fields $-v l^{a}$ and $w n^{a}$ on $j^{-}\left(\mathscr{I}^{+}\right)$and $j^{+}\left(\mathscr{I}^{-}\right)$ respectively. The solution determined by the Cauchy data will therefore admit an isometry group $\theta_{u}$ whose Killing vector $\hat{K}^{a}$ will coincide with $-v l^{a}$ or $w n^{a}$ on the horizons. 
In the case of the Einstein equations with a scalar field, the additional Cauchy data needed is the value of the field on the two horizons. As $\Phi_{00}$ and $\Phi_{22}$ are zero, the field must be constant along each generator. The Cauchy data is thus invariant under the transformation $\theta_{u}$ in this case also. For the Einstein-Maxwell equations the additional data is $F_{a b} l^{a} m^{b}$ on $\dot{J}^{-}\left(\mathscr{I}^{+}\right), F_{a b} n^{a} \bar{m}^{b}$ on $\dot{J}^{+}\left(\mathscr{I}^{-}\right)$and $F_{a b}\left(l^{a} n^{b}+m^{a} \bar{m}^{b}\right)$ on $\mathscr{F}$. However the first two are zero as $\Phi_{00}$ and $\Phi_{22}$ are zero and the third is invariant under $\theta_{u}$. Similar results will hold for other well behaved fields.

The Killing vector $K^{a}$ will be tangent to the horizons since they are invariants under the isometry $\phi_{t}$. Thus on the horizons it will either be directed along the null generators or it will be spacelike. The former will be the situation if the solution is not only stationary but static [17]. In this case the Killing vectors $K^{a}$ and $\hat{K}^{a}$ will coincide. However one can appeal to Israel's results $[8,18]$ to show that the solution must be spherically symmetric if it is either empty or contains only a Maxwell field.

If the solution is not static but only stationary and is empty, one can generalise a result of Lichnerowitz [19] to show that there will be a region where $K^{a}$ is spacelike. (Details will be given in [3].) Part, at least, of this region will be outside the horizon. If the horizon is contained in this region, the Killing vectors $K^{a}$ and $\hat{K}^{a}$ will be distinct and so the solution will be axisymmetric. A particle travelling along a null geodesic generator of the horizon would be moving with respect to the stationary frame, i.e. the integral curves of $K^{a}$. Thus, in a sense, the horizon would be rotating with respect to infinity. One can therefore say that any rotating black hole which satisfies conditions (i)-(v) must be axisymmetric.

There remains the possibility that there could be stationary, nonstatic solutions in which the horizon was not rotating but in which $K^{a}$ was spacelike in a region outside and disjoint from the horizon. In such a situation there would have to be neutral points outside the horizon where $K^{a}$ was timelike and the gradient of $K^{a} K_{a}$ was zero. I have not been able to rule out this possibility but it seems to be unstable in that one could extract an indefinitely large amount of energy by a method proposed by Penrose [20]: consider a small particle with momentum $p_{1}^{a}=m_{1} v_{1}^{a}$ where $m_{1}$ is the mass and $v_{1}^{a}$ is the future directed unit tangent vector to the world-line. If the particle moves on a geodesic its energy $E_{1}=p_{1}^{a} K_{a}$ will be constant. Suppose that the particle were to fall from infinity into the region where $K^{a}$ was spacelike and there divided into two particles momenta $p_{2}^{a}$ and $p_{3}^{a}$. By local conservation, $p_{1}^{a}=p_{2}^{a}+p_{3}^{a}$. Since $K^{a}$ was spacelike, one could arrange that $E_{2}=p_{2}^{a} K_{a}$ was negative. Then $E_{3}>E_{1}$ and particle 3 could escape to infinity, where its total energy (rest-mass plus kinetic) would be greater than that of the original particle. Particle 2 on the other hand would have to remain in the region 
where $K^{a}$ was spacelike and so could neither escape to infinity nor fall through the horizon. By repeating this process many times one could gradually extract energy from the solution. As one did this the solution would presumably change gradually. However the region where $K^{a}$ is spacelike could not disappear since there would have to be somewhere for the negative energy particles to go. If it remained disjoint from the horizon, one would apparently extract an indefinite amount of energy. If, on the other hand, the region moved so that it included the horizon, $K^{a}$ and $\hat{K}^{a}$ would be distinct and so, suddenly, the solution would be axisymmetric. I therefore feel that such a situation will not occur and that any stationary, nonstatic, black hole will be axisymmetric.

It is worth noting that the field equations did not play a very important part in the above argument. Thus one can apply it also to the case of a rotating black hole surrounded by a ring of matter. The solution will not be analytic at the ring but it will be elsewhere, and so the above result will still hold. At first sight this seems to lead to a paradox. Consider a rotating star surrounded by a non-rotating square frame of rods. Suppose that the star collapsed to produce a rotating black hole. From the above it appears that the solution ought to become axisymmetric. However the presence of the square frame will prevent this.

The answer seems to be that the field of the frame will distort the rotating black hole slightly. The reaction of the rotating black hole back on the frame will cause it to start rotating and so to radiate gravitational waves. Eventually the rotation of both the black hole and the frame will be damped out and the solution will approach a static state in which $K^{a}$ and $\hat{K}^{a}$ coincide and which therefore does not have to be axisymmetric. I am grateful to James B. Hartle and Brandon Carter for suggesting this solution to the paradox.

\section{The Topology of the Event Horizon}

In this section I shall prove that each connected component of the 2 -surface $\mathscr{L}(t)$ in the event horizon has the topology $S^{2}$. This is done by showing that, if it had any other topology, one could deform it outwards into the exterior region in such a way that the future directed outgoing null geodesics orthogonal to it would be converging. As in Section 2, this would lead to a contradiction.

Let $l^{a}$ be the future directed null vector tangent to the future event horizon and let $n^{a}$ be the other future directed null vector orthogonal to $\mathscr{L}(t)$ normalized so that $l^{a} n_{a}=1$ but with the freedom $l^{a} \rightarrow e^{a} l^{a}, n^{a} \rightarrow e^{-a} n^{a}$ not restricted for the moment. Now move each point of $\mathscr{L}(t)$ an affine distance $-w$ along the null geodesics with tangent vector $n^{a}$. This moves $\mathscr{L}(t)$ to the past into $J^{-}\left(\mathscr{I}^{+}\right)$. To keep $l^{a}$ orthogonal to the 2-surface, it 
has to be propagated so that

$$
\tau=\bar{\alpha}+\beta
$$

where $\tau=l_{a ; b} m^{a} n^{b}$. The change of the convergence $\varrho$ produced by this movement is given by one of the Newman-Penrose equations [13]:

$$
\frac{d}{d w} \varrho=\bar{\delta} \tau+(\bar{\beta}-\alpha) \tau-\bar{\tau} \tau-\psi_{2}-2 \Lambda
$$

where $\Lambda=R / 24, \alpha-\bar{\beta}=\bar{m}_{a ; b} m^{a} \bar{m}^{b}$ and $\bar{\delta} \tau=\bar{m}^{a} \tau_{; a}$. The combination $\bar{\delta}-(\alpha-\bar{\beta})$ acting on the spin weight +1 quantity $\tau$ is the covariant Newman-Penrose operator $\bar{\gamma}[21]$.

Under a rescaling transformation $l^{\prime a}=e^{a} l^{a}, n^{\prime a}=e^{-a} n^{a}$, the quantity $\tau$ changes to $\tau^{\prime}=\tau+\delta a$ and so $\frac{d}{d w} \varrho$ changes to

$$
\frac{d}{d w^{\prime}} \varrho^{\prime}=-\tau^{\prime} \bar{\tau}^{\prime}+\bar{\gamma} \tau+\bar{\gamma} \delta a-\psi_{2}-2 \Lambda \text {. }
$$

The term $\bar{\gamma} \delta a$ is the Laplacian of $a$ in the 2-surface. One can choose $a$ so that the sum of the last four terms on the right of Eq. (2) is constant on $\mathscr{L}(t)$. The sign of this constant value will be determined by that of the integral of $-\left(\psi_{2}+2 \Lambda\right)$ over $\mathscr{L}(t)(\bar{\gamma} \tau$, being a gradient, has zero integral). This integral can be evaluated from another Newman-Penrose equation which can be written as

$$
\gamma(\alpha+\bar{\beta})-\bar{\gamma}(\bar{\alpha}+\beta)+\gamma(\alpha-\bar{\beta})+\bar{\gamma}(\bar{\alpha}-\beta)=-2 \psi_{2}+2 \Lambda+2 \Phi_{11}
$$

where $\Phi_{11}=-\frac{1}{4} R_{a b}\left(l^{a} n^{b}+m^{a} \bar{m}^{b}\right)$. When integrated over the 2-surface the terms in $\bar{\alpha}+\beta$ disappear but there is in general a contribution from the $\bar{\alpha}-\beta$ terms because the vector field $m^{a}$ will have singularities on the 2 -surface. The contribution from these singularities is determined by the Euler number $\chi$ of the 2-surface. Thus

$$
\int\left(-\psi_{2}+\Phi_{11}+\Lambda\right) d S=\pi \chi .
$$

(The real part of this equation is, in fact, the Gauss-Bonnet theorem.) Therefore

$$
-\int\left(\psi_{2}+2 \Lambda\right) d S=\pi \chi-\int\left(\Phi_{11}+3 \Lambda\right) d S
$$

For the electromagnetic and other reasonable matter fields, obeying the Dominant Energy condition [25] $\Phi_{11}+3 \Lambda \geqq 0$. The Euler number $\chi$ is +2 for the sphere, 0 for the torus and negative for other compact orientable 2-surfaces. ( $\mathscr{L}(t)$ has to be orientable as it is a boundary.)

Suppose that the right hand side of Eq. (5) was negative. Then one could choose $a$ so that $\frac{d}{d w^{\prime}} \varrho^{\prime}$ was negative everywhere on $\mathscr{L}(t)$. For a 
small negative value of $w^{\prime}$ one would obtain a 2-surface in $J^{-}\left(\mathscr{I}^{+}\right)$such that the outgoing null geodesics orthogonal to the surface were converging. This would lead to a contradiction.

Suppose now that $\chi$ was zero and that $\Phi_{11}+3 \Lambda$ was zero on the horizon. Then one could choose a so that the sum of the last four terms in Eq. (2) was zero everywhere on $\mathscr{L}(t)$. If $\psi_{2}$ was nonzero somewhere on $\mathscr{L}(t)$, the term $-\tau^{\prime} \bar{\tau}^{\prime}$ in Eq. (2) would be nonzero and one could change a slightly so as to make $\frac{d}{d w^{\prime}} \varrho^{\prime}$ negative everywhere on $\mathscr{L}(t)$. This would again lead to a contradiction. If, on the other hand, $\psi_{2}$ was zero everywhere on $\mathscr{L}(t)$, the Cauchy data would be zero and so the solution would be flat. One could not have a flat toroidal horizon in a flat space which is Euclidean at infinity.

This shows that $\mathscr{L}(t)$ and hence $\mathscr{F}$ has spherical topology. It has not been excluded that $\mathscr{L}(t)$ might have several disconnected components, each topologically spheres, which represent separate black holes at constant distance from each other along the axis of symmetry. For reasons explained in the next section I think this could occur only in the limiting case where the black holes have a charge $q= \pm G^{\frac{1}{2}} m$.

\section{Energy Limits}

In view of the previous two sections, it seems reasonable to assume that in a gravitational collapse the solution outside the horizon settles down into a Kerr solution with $a^{2}<m^{2}$. If the collapsing body had a net electrical charge of $q$ e.s.u., one would expect the solution to settle down to a charged Kerr solution [10] with $a^{2}+e^{2}<m^{2}$ where $e=G^{-\frac{1}{2}} q$. One would therefore expect the area of the 2 -surface $\partial \mathscr{B}(t)$ in the horizon to approach the area of a 2-surface in the horizon of the Kerr solution. This area is

$$
4 \pi G^{2} c^{-4}\left(2 m^{2}-e^{2}+2 m\left(m^{2}-a^{2}-e^{2}\right)^{\frac{1}{2}}\right) .
$$

Consider a black hole which by a surface $\mathscr{S}(t)$ has settled down to a Kerr solution with parameters $m_{1}, a_{1}$ and $e_{1}$. Suppose the black hole now interacts with various particles or fields and then settles down again by a surface $\mathscr{S}\left(t^{\prime}\right)$ to a Kerr solution with parameters $m_{2}, a_{2}$ and $e_{2}$. From Section 2 it follows that the area of $\partial \mathscr{B}\left(t^{\prime}\right)$ is greater than or equal to the area of $\partial \mathscr{B}(t)$. In fact it must be strictly greater if there is any disturbance at the event horizon. Thus

$$
2 m_{2}^{2}-e_{2}^{2}+2 m_{2}\left(m_{2}^{2}-a_{2}^{2}-e_{2}^{2}\right)^{\frac{1}{2}}>2 m_{1}^{2}-e_{1}^{2}+2 m_{1}\left(m_{1}^{2}-a_{1}^{2}-e_{1}^{2}\right)^{\frac{1}{2}} .
$$

Note that $m_{2}$ can be less than $m_{1}$ if $a_{1}$ or $e_{1}$ are nonzero. One can interpret this as meaning that one can extract rotational or electrostatic energy 
from a black hole. One way of extracting rotational energy would be to construct an asymmetric frame of rods around the black hole. According to the argument in Section 3, the frame would start rotating and would emit gravitational waves until the rotation of the black hole was damped out. Another way of extracting energy would be to use the fact that the Killing vector $K^{a}$ is spacelike on and in a region just outside the event horizon. One could then employ the procedure described in Section 3 of sending in a particle and getting one back with greater energy. In this case the negative energy particles could fall through the horizon. Christodoulou [22] has shown that using this process one can get arbitrarily near the limit set by inequality (7). Using charged particles one can extract electrostatic energy from a black hole. By lowering a particle of the opposite charge down the axis on a rope one can get arbitrarily near the limit set by (7).

Consider now a situation in which two stars a long way apart collapse to form black holes $\mathscr{B}_{1}(t)$ and $\mathscr{B}_{2}(t)$. One can neglect the interaction between them and take the areas of $\partial \mathscr{B}_{1}(t)$ and $\partial \mathscr{B}_{2}(t)$ to be given by formula (6) with the values of the parameters $m_{1}, a_{1}, e_{1}$ and $m_{2}, a_{2}, e_{2}$ respectively. Suppose the two black holes now collide and merge to form a single black hole $\mathscr{B}_{3}\left(t^{\prime}\right)$. This process will give rise to a certain amount of gravitational radiation and, if the black holes are charged, of electromagnetic radiation as well. By the conservation law for weakly asymptotically simple space-times [23], the energy of this radiation is $\left(m_{1}+m_{2}\right.$ $\left.-m_{3}\right) c^{2}$. This is limited by the requirement that the area of $\partial \mathscr{B}_{3}\left(t^{\prime}\right)$ must be greater than the sum of the areas of $\partial \mathscr{B}_{1}(t)$ and $\partial \mathscr{B}_{2}(t)$. This gives the inequality

$$
\begin{gathered}
2 m_{3}^{2}-e_{3}^{2}+2 m_{3}\left(m_{3}^{2}-a_{3}^{2}-e_{3}^{2}\right)^{\frac{1}{2}} \\
>2 m_{2}^{2}-e_{2}^{2}+2 m_{2}\left(m_{2}^{2}-a_{2}^{2}-e_{2}^{2}\right)^{\frac{1}{2}}+2 m_{1}^{2}-e_{1}^{2}+2 m_{1}\left(m_{1}^{2}-a_{1}^{2}-e_{1}^{2}\right)^{\frac{1}{2}} .
\end{gathered}
$$

The fraction $\varepsilon=\left(m_{1}+m_{2}\right)^{-1}\left(m_{1}+m_{2}-m_{3}\right)$ of energy radiated is always less than $1-2^{-\frac{3}{2}}$. If $e_{1}$ and $e_{2}$ are zero or have the same sign, then $\varepsilon<\frac{1}{2}$. If, in addition, $a_{1}=a_{2}=0$, then $\varepsilon<1-2^{-\frac{1}{2}}$.

By the conservation of charge, $e_{3}=e_{1}+e_{2}$. Angular momentum, on the other hand, can be carried off by the radiation. This cannot happen however if the situation is axisymmetric, i.e. if the black holes have their rotation axes aligned along their direction of approach to each other. Then $m_{3} a_{3}=m_{1} a_{1}+m_{2} a_{2}$. It can be seen that if the angular momenta have the same sign there is less energy available to be radiated than if they have opposite signs. This suggests that there may be a spin dependent force between two black holes as indeed one might expect from the analogy between angular momentum and magnetic dipole moment. Unlike the electrodynamic case, the force would be attractive if the 
angular momenta had opposite directions and repulsive if they had the same direction. However, even in the limiting case $m_{1}=m_{2}=a_{1}=a_{2}$, it is still possible to radiate some energy. This suggests that the repulsive force is never strong enough to balance the attractive force between the masses. It seems that the only way to obtain a strong enough repulsive force is to go to the other limiting case in which $m_{1}=e_{1}, m_{2}=e_{2}$ and $a_{1}=a_{2}=0$. Hartle and I [24] have found static solutions containing two or more such black holes.

Acknowledgment. I have been greatly helped by discussions with Brandon Carter, George Ellis, James Hartle and Roger Penrose.

\section{References}

1. Penrose, R.: Phys. Rev. Letters 14, 57 (1965).

2. - In: de Witt, C.M., Wheeler,J.A. (Eds.): Battelle Rencontres (1967). New York: Benjamın 1968.

3. Hawking, S.W., Ellis, G.F.R.: The large scale structure of space time. Cambridge: Cambridge University Press (to be published).

4. - Penrose, R.: Proc. Roy. Soc. A 314, 529 (1970).

5. Penrose, R.: Seminar at Cambridge University, January 1971 (unpublished).

6. Gibbons, G. W., Penrose, R.: To be published.

7. Geroch, R.P.: J. Math. Phys. 11, 437 (1970).

8. Israel, W.: Phys. Rev. 164, 1776 (1967).

9. Carter, B.: Phys. Rev. Letters 26, 331 (1971).

10. - Phys. Rev. 174, 1559 (1968).

11. Müller zum Hagen, H.: Proc. Cambrıdge Phil. Soc. 68, 199 (1970).

12. Carter, B.: Commun. math. Phys. 17, 233 (1970).

13. Newman, E.T., Penrose, R.: J. Math. Phys. 3, 566 (1962).

14. Hawking, S. W.: Proc. Roy. Soc. A 300, 187 (1967).

15. Sachs, R. K.: J. Math. Phys. 3, 908 (1962).

16. Penrose, R.: Characteristic initial data for zero rest mass including gravitation, preprint (1961).

17. Carter, B.: J. Math. Phys. 10, 70 (1969).

18. Israel, W.: Commun. math. Phys. 8, 245 (1968).

19. Lichnerowicz, A.: Théories relativistes de la gravitation et de l'électromagnétisme. Paris: Masson 1955.

20. Penrose, R.: Nuovo Cimento Serie 1, 1, 252 (1969).

21. Newman, E.T., Penrose, R.: J. Math. Phys. 7, 863 (1966).

22. Christodoulou, D.: Phys. Rev. Letters 25, 1596 (1970).

23. Penrose, R.: Phys. Rev. Letters 10, 66 (1963).

24. Hartle, J. B., Hawking, S. W.: Commun. math. Phys. To be published.

25. Hawking, S. W.: Commun. math. Phys. 18, 301 (1970).

S. W. Hawking

Institute of Theoretical Astronomy

University of Cambridge

Madingley Road

Cambridge, England 